\section{PSICOLOGÍA IBEROAMERICANA}

\section{Psicología lberoamericana}

ISSN: 1405-0943

psicología.iberoamericana@uia.mx

Universidad Iberoamericana, Ciudad de

México

México

Mendoza González, Brenda

Bullying entre Pares y el Escalamiento de Agresión en la Relación Profesor-Alumno

Psicología Iberoamericana, vol. 19, núm. 1, enero-junio, 2011, pp. 58-71

Universidad Iberoamericana, Ciudad de México

Distrito Federal, México

Disponible en: http://www.redalyc.org/articulo.oa?id=133920896007

Cómo citar el artículo

Número completo

- Más información del artículo

Página de la revista en redalyc.org

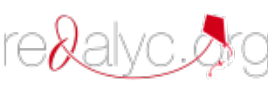

Sistema de Información Científica

Red de Revistas Científicas de América Latina, el Caribe, España y Portugal

Proyecto académico sin fines de lucro, desarrollado bajo la iniciativa de acceso abierto 


\title{
Bullying entre Pares y el Escalamiento de Agresión en la Relación Profesor-Alumno
}

\author{
Peer Bullying and Aggressive Escalations in the Student-Teacher Relationship
}

Brenda Mendoza González*

Secretaria de Educación Pública, México

\section{RESUMEN}

El trabajo explora la relación entre el bullying entre pares, y entre alumnado y profesorado, ambos medidos desde la percepción del alumnado. Participaron 757 estudiantes de secundaria de escuelas públicas de Madrid. Se describen los tipos de adolescentes que participan en episodios de bullying entre pares (bullies, víctimas y no involucrados), y bullying entre profesorado (desafección, acoso, neutro). La relación identificada demuestra otra cara de la violencia y el bullying, demostrándose que el 51\% de los estudiantes relacionados con escalamiento agresivo con el profesorado, también se involucra en situaciones de bullying en la relación con sus compañeros; detectándose que los estudiantes perciben un proceso de reciprocidad entre maltrato que reciben y ejercen hacia los profesores, proceso que facilita la escalamiento de agresión entre éstos y los alumnos.

Descriptores: bullying, agresión, reciprocidad coercitiva, escalamiento, maltrato profesorado-alumnado.

\section{ABSTRACT}

This work explores the relationship between bullying among students and problems with teachers, both assessed from the students' point of view. Participants were 757 adolescents from public secondary schools in Madrid, Spain. We detected two problems with teachers differing in their extent and seriousness. In both cases the adolescents perceive reciprocity between the aversive treatment they receive from teachers and the way they themselves treat them. The most widespread problem consists in a lack of opportunities, demotivation and disruptive behaviour. The most serious problem appears to involve an aggressive escalation. We found a relationship between these two faces of school violence, reflected in the fact that $51 \%$ of students involved in aggressive escalations with teachers also acknowledge bullying their schoolmates.

Key words: bullying, aggression, students, problems with teachers, disruption 


\section{INTRODUCCIÓN}

La violencia y acoso escolar son fenómenos viejos; ambos son subtipos de agresión, por lo tanto, el bullying es violencia; sin embargo, la violencia escolar no se considera bullying, ya que existen características definitorias e éste, que al identificarlas permiten guiar las estrategias de atención.

El estudio del bullying comenzó en la década de los setenta, convirtiéndose en objeto de investigación en Noruega; Dan Olweus fue el primero en describirlo, determinar su incidencia y crear programas de intervención (Olweus, 1978). El bullying o su traducción en castellano como acoso escolar (Olweus, 2005) es un tipo de agresión que incluye conductas de maltrato físico, emocional, sexual, que se repite y prolonga a lo largo del tiempo; existiendo una relación de desequilibrio de poder entre el acosador y la víctima.

Las investigaciones internacionales señalan que el tipo de bullying que con mayor frecuencia se presenta es la exclusión (no dejar participar a los compañeros en grupos de trabajo o lúdicos), escalando a agresión verbal, a la física, y en algunos casos puede llegar a niveles extremos, como abuso sexual, y uso de armas. Por lo que pueden desarrollarse estrategias de detección temprana comenzando por identificar a alumnos que son ignorados, que reciben burlas constantemente de los compañeros al participar en clase, así como al alumnado que solicita con frecuencia al profesorado trabajar solo (por alguna razón no trabaja en equipo).

Conviene destacar que el bullying no es una conducta que "surge azarosamente", ya que el agresor ha aprendido que maltratar a un compañero "le trae ganancias" a bajo costo, es decir, ocasionalmente recibe consecuencias negativas y generalmente obtiene lo que desea; el alumno agresor aprovecha las ocasiones en las que el profesorado confunde al bullying como conductas normales o que son parte de un juego (i.e. llamarse por apodos, "jugar a darse zapes”, esconder las propiedades de otros, entre muchas otras).

El comportamiento de bullying no es una conducta que se consolida "de un día para otro", ya que el alumno durante algún tiempo y en diversos escenarios (i.e. escuela, familia) aprende a usarla en el día a día para lograr lo que desea (i.e. poder, control, dinero, etc.), y en este proceso de aprendizaje se presentan eventos ambientales que facilitan que el alumno la use, es decir se le refuerza el uso de la conducta de bullying, debido a que generalmente su comportamiento no tiene consecuencias (i.e. el profesorado no lo castiga, e incluso en algunos casos sí lo hace con la víctima), y con el paso del tiempo los niños, adolescentes, jóvenes aprenden el valor funcional del comportamiento de bullying, por lo que extienden su uso a lo largo del tiempo (i.e. primaria, secundaria, bachiller) (Mendoza \& Santoyo, 2010).

\section{Tipos de alumnos que participan en bullying}

Tradicionalmente en las investigaciones de acoso escolar, los escolares se han clasificado como agresores, víctimas y espectadores. El análisis de Salmivalli, Lagerspetz, Bjórkqvist, Österman y Kaukiainen (1996), ofrece una muestra clara de esta tipología, identificando, a través del estudio, que no sólo los acosadores y sus víctimas influyen en el acoso escolar, sino también otros compañeros de la clase debido a sus acciones o ausencia de ellas. De acuerdo con este estudio, el 87\% de los escolares ha asumido uno de los diferentes roles que existen en el acoso escolar, ya que existen los que establecen alianza con los agresores y se convierten en sus asistentes o secuaces, hay otros que se alinean con las víctimas y tratan de defenderlas, y por último están los testigos o mirones. Sin embargo, la evidencia empírica de otras investigaciones ha demostrado que los papeles de agresor, víctima y espectador permanecen uno o dos años (Boulton \& Smith, 1994; Salmivalli, Lappalainen \& Lagerspetz, 1998).

Actualmente existe evidencia empírica que señala que los tipos de estudiantes involucrados en la violencia escolar son: agresor puro, víctima pura, espectador y otro tipo de estudiante que participa con un doble rol: víctima/bully (Austin \& Joseph, 1996; Boulton \& Smith, 1994; Salmivalli, et al., 1996; Slee \& Rigby, 1993; Unnever, 2005). Por su parte, Eslea, Menesini, Morita, O'Moore, Mora-Merchán, Pereira, Smith y Zhang (2003), identifican a un estudiante más: el alumno neutral, definido como el que no se involucra en situaciones de acoso escolar o bullying.

Ireland y Power (2004), definen a la víctima escolar como aquellos estudiantes que únicamente expresan recibir conductas agresivas de otros; al agresor escolar como aquellos que únicamente ejercen conductas de 
agresión hacia otros; a la víctima/agresor como los que reportan conductas indicativas de acosar a otros y de ser acosados por otros, y finalmente, definen a los "no involucrados" como estudiantes que no participan ni como víctimas, ni como agresores.

En una reunión internacional organizada por el Centro Reina Sofía para estudios de violencia, Olweus (2005) presentó los roles de los participantes en el ciclo del bullying, expresando que existen ocho participantes: bully o bullies; seguidor o secuaz; seguidor (bully pasivo), seguidor pasivo (probable bully), testigo no implicado, posible defensor, víctima y defensor de víctima.

Muchos han sido los esfuerzos para identificar las características del alumnado participante en este tipo de situaciones. Con respecto al bully, se ha identificado que es un estudiante que abusa de su fuerza para dominar a otros (suelen ser físicamente más fuertes que los demás), tiene dificultad para ponerse en el lugar de los otros (empatía) (Olweus, 1993), exhibe actitudes intolerantes y elevada justificación de la violencia (Mendoza, 2006), así como comportamiento impulsivo, baja tolerancia a la frustración; dificultad para cumplir normas y comportamiento disruptivo (Kokkinos \& Panayitou, 2004), interactúa negativamente con el profesorado y presenta problemas académicos (Natvig, Albrektsen \& Qvarnstrom, 2001; Ma, 2002). Este tipo de estudiantes son percibidos por sus compañeros como intolerantes y arrogantes, y al mismo como que se sienten fracasados. La mayoría de los estudios encuentra que entre los bullies hay más hombres que mujeres (Defensor del Pueblo, 2007), identificándose que una de las principales condiciones que lleva a ejercer la violencia es la identificación con el dominio de los demás, asociada al estereotipo masculino tradicional: "machismo" (Young \& Sweeting, 2004). Los bullies tienen una fuerte orientación a dominar a los demás, cuyo comportamiento les funciona para obtener poder, comunicación y protagonismo, que no obtienen al exhibir un comportamiento distinto. Cabe plantear como hipótesis que estos problemas podrían llevarles también a establecer una relación coercitiva, de confrontación de poder, con determinados profesores, aunque esto ha sido muy poco investigado.

Con respecto al alumnado víctima, se ha identificado que se encuentra en mayor riesgo de serlo cuan- do tiene escasas o nulas relaciones sociales; son tímidos, tienen alguna incapacidad o sobreprotección maternal (Hawker \& Boulton, 2000; Monks \& Smith, 2000), presentan déficits en habilidades asertivas (Kokkinos \& Panayouotu, 2004), exhiben una conducta muy pasiva ante situaciones violentas, elevada inseguridad y baja autoestima (Olweus, 2001); la víctima es un estudiante hombre o mujer que se identifica con el estereotipo femenino tradicional (Mendoza, 2006).

El alumnado que desempeña doble rol: víctimabully, es un tipo de víctima más reactiva, impulsiva, que exhibe incluso comportamiento antisocial (Olweus, 2001). Se distingue por tener muy baja aceptación social y pocas habilidades para solucionar problemas (Andreou, 2001); son escolares que se encuentran en circunstancias de riesgo sociales y familiares (Monks \& Smith, 2000).

El alumnado que no se involucra en situaciones de bullying se caracteriza por ser un estudiante que no justifica el uso de la violencia, acepta la diversidad que exhiben sus compañeros de clase y exhibe tolerancia ante ellas, no es racista, sexista, y al parecer ha superado la dicotomía sexista, es decir, no se identifica con el estereotipo femenino o masculino tradicional, ya que incorpora la totalidad de los valores y comportamientos, tanto masculinos como femeninos inmersos en la sociedad (Mendoza, 2006).

\section{Maltrato en la interacción profesorado- alumnado}

Wilson y Herrnestein (1985) predicen que los niños coercitivos en el contexto escolar, muestran una baja preferencia por la conducta académica, ya que el valor relativo motivacional de la conducta agresiva exhibida es mayor al de su conducta académica. Dicho planteamiento ha sido comprobado empíricamente en estudios en los que se ha identificado que los niños que exhiben comportamiento agresivo se encuentran menos motivados para desarrollar tareas escolares (Santoyo, Colmenares, Figueroa, Cruz, \& López, 2008). Esta predicción ha sido comprobada también con estudios de bullying (Mendoza \& Santoyo, 2008), dicho riesgo disminuye cuando el alumnado tiene la percepción de recibir un apoyo por parte del profesorado (Natvig, et al., 2001). También se ha observado que los bullies y los bullies-víctima tienen más problemas de compor- 
tamiento disruptivo que sus compañeros (Kokkinos \& Panayiotou, 2004); de hecho se ha encontrado que cuando el profesorado mantiene expectativas positivas sobre el rendimiento del alumnado se relaciona con menores tasas de bullying en la adolescencia, mientras que en edades anteriores las variables más relevantes, en este sentido, son la participación escolar de las familias y la calidad de la disciplina (Ma, 2002).

Los estudios realizados sobre el comportamiento disruptivo reflejan que el profesorado dirige hacia el alumnado más críticas y menos comentarios positivos (Reynard \& Sonuga-Barke, 2005), conductas que suelen reducir la motivación de los alumnos por el aprendizaje y el rendimiento (Wentzel, 2002). Cuando se pregunta a los alumnos tienden a atribuir su propio comportamiento disruptivo a falta de apoyo y de reconocimiento por parte del profesor (Bru, Stephens \& Tosheim, 2002); por su parte, el profesorado identifica que emplea prácticas de disciplina coercitivas ante el comportamiento disruptivo del alumnado (Lewis, 2001). La reacción coercitiva del profesor y la disrupción de los alumnos podrían así formar parte de una escalada coercitiva similar a la observada en contextos familiares (Patterson, 1982) que podría, en determinadas condiciones, ser el origen de conductas instrumentales de características similares al acoso, tanto por parte del profesor como por parte de los alumnos. Con respecto al acoso en la relación profesoradoalumnado, hace más de 10 años Olweus (1993), identificó que el $2 \%$ del alumnado encuestado informaba recibir malos tratos por parte del profesorado, señalándose con ello a la primer investigación sensible al tema del maltrato del profesor hacia el alumno. Años más tarde este autor desarrolló otra investigación con una muestra de 5100 profesores noruegos en escuelas elementales y bachillerato, detectando que el 10\% exhibía maltrato hacia uno o más alumnos (Olweus, 1999).

En este sentido, Benbeneshty, Zeira y Astor (2002), han encontrado que la cuarta parte de los estudiantes de $7^{\circ}$ a $11^{\circ}$ curso evaluados a través del auto-informe en una muestra representativa de Israel declaran haber sido maltratados emocionalmente por algún miembro del equipo educativo; y el $18.7 \%$ dice haber sufrido alguna forma de maltrato físico. El hecho de ser maltratados por parte del profesor puede originar desanimo y pasividad, como se había descrito hace tiempo (Brophy, 1985), o comportamiento disruptivo e incluso violencia en quienes lo padecen (Hyman \& Perone, 1998),; violencia que podrían dirigir hacia los profesores o hacia los compañeros.

Son escasos los estudios científicos realizados sobre el bullying entre profesores y alumnos, en el Reino Unido Terry (1998) encontró que el 9.9\% de los profesores de secundaria evaluados afirmó haber sufrido bullying por parte de sus alumnos, confirmándose la hipótesis según la cual los profesores novatos padecen dicha situación con mayor frecuencia que los otros, sin embargo, no se identificaron diferencias en el género del profesorado en su posición de víctimas. El 9.9\% profesorado afirmó que conocen a profesores que reciben bullying de sus alumnos, y el $51.4 \%$ reconocer que por lo menos uno de sus compañeros lo dirige hacia sus alumnos.

En Australia, Delfabbro, Winefield, Trainor, Dollard, Anderson, Metzer y Hammarstrom (2006), realizaron una investigación para conocer la relación de bullying entre profesorado y alumnado. Sus resultados reflejan que el alumnado victimizado por sus pares muestran un funcionamiento psicológico pobre, baja auto estima y baja auto imagen, mientras que los estudiantes víctimas de bullying del profesorado tienen menor capacidad académica (evaluada a través de las expectativas de los profesores), más probabilidad de implicarse en conductas de riesgo (i.e. uso de drogas) y una menor intención de acabar la escuela (condición que incrementa en cinco veces la probabilidad de ser víctima del bullying del profesor). Estos resultados reflejan que existe una clara relación entre falta de identificación con lo académico y riesgo de victimización por parte del profesor, pero no permiten conocer cuál es el punto de vista de estos alumnos sobre su comportamiento disruptivo, y hasta qué limite lo llevan, ni si lo reconocen de naturaleza diferente o similar al maltrato que afirman recibir. Para responder a estos interrogantes es preciso estudiar el punto de vista de los estudiantes sobre su participación en los papeles del acoso escolar -agresor, víctima, espectador y víctima-bully, y en las dos relaciones que se producen en el aula -entre compañeros y con profesores-. El objetivo general del trabajo que aquí se presenta está basado en el punto de vista de los estudiantes, que pretende de forma específica: 
1) Delimitar las principales situaciones respecto a la participación en bullying entre iguales, considerando tanto el papel de víctima como el de acosador (bully), e incluyendo conductas de distinta naturaleza: exclusión, agresiones de gravedad media y de gravedad extrema.

2) Delimitar las principales situaciones respecto a la participación en los problemas de interacción que surgen con los profesores, en las que la víctima pueda ser el alumno y en las que pueda serlo el profesor, e incluyendo problemas de falta de vinculación, rechazo, discriminación y agresiones de distinto nivel de gravedad.

3) Comprender qué relación existe entre el trato que declaran recibir y ejercer en su interacción con los profesores, en las situaciones mencionadas en el objetivo anterior.

4) Conocer qué relación existe entre el papel que los adolescentes reconocen desempeñar en situaciones de bullying entre iguales y en los problemas de interacción con los profesores que se detecten al emplear una metodología similar.

En esta investigación se busca ofrecer información que no se limite a describir e identificar variables que influyen en el proceso de aprendizaje del bullying entre pares, si no que desde una perspectiva integral se explorará el proceso de reciprocidad coercitiva entre profesorado y alumnado, así como su relación con la participación de este último como bully o víctima en episodios de bullying entre compañeros, episodios que innegablemente afectan la calidad educativa.

\section{MÉTODO}

\section{Participantes}

Participaron 757 sujetos (54\% varones y $46 \%$ mujeres), con edades comprendidas entre 12 y 18 años de edad, $75 \%$ españoles y $25 \%$ extranjeros. Los sujetos estaban inscritos en tres institutos de educación secundaria obligatoria (ESO) de Madrid (zona norte, centro, y sur).

\section{Instrumentos}

En esta investigación se han utilizado dos instrumentos de evaluación:
1. Cuestionario sobre la violencia entre iguales en la escuela (Díaz-Aguado, Martínez Arias y Martín Seoane, 2004)), derivado del utilizado en el estudio sobre la violencia escolar del Defensor del Pueblo (2007), que sigue la misma metodología utilizada desde Olweus (1978) para la evaluación del bullying en iguales. Consiste en preguntar por la frecuencia (nunca, alguna vez, a veces, a menudo) con la que se han sufrido como víctima o ejercido como agresor una serie de 15 situaciones, que van desde el aislamiento hasta las agresiones con armas. El coeficiente alpha de crombach, para el total de la prueba, es de .75 Consta de dos escalas en función del papel desempeñado en el bullying. La escala de victimización incluye tres factores: exclusión ("Mis compañeros me ignoran," "hablan mal de mí"...); 2) víctimizacion de gravedad media ("me roban cosas," "me pegan," "me llaman por motes que me ofenden"); 3) victimización de gravedad extrema ("me amenazan con armas," "me obligan con amenazas a situaciones de carácter sexual," "me obligan a hacer cosas que no quiero con amenazas"...). La escala de agresión consta de dos factores: 1) exclusión y agresión de gravedad media "rechazar", insultar, "llamar por motes que ofenden", "esconder cosas"; 2) y agresión grave (obligándole a hacer cosas que no quiere con amenazas, robándole cosas, amenazándole con armas, pegándole, amenazándole para meterle miedo"...).

2. Cuestionario de Maltrato en la Interacción profesor-alumno (Mendoza, 2006), que se ha elaborado adaptando la metodología empleada en los estudios sobre bullying a la peculiaridad de los problemas que se producen en las relaciones entre alumnos y profesores. Incluye dos escalas: sobre la victimización que sufre el alumno y la que ejerce sobre el profesor. La primera escala consta de 16 situaciones que, según el análisis factorial, se agrupan en cuatro factores: 1) trato discriminatorio ("el profesor me tiene manía", "me regaña más que a otros aún cuando mi comportamiento es parecido"; "me amenaza", "me interrumpe cuando hablo", "me grita"); 2) agresión ("me ha agredido físicamente", "ha roto alguna de mis cosas", me tira objetos", "me insulta"); 3 ) exclusión ("me 
ignora", "me impide participar", "me rechaza"; 4) maltrato emocional, ("me ridiculiza", "se burla de mí"; "me llama por motes que me ofenden". El coeficiente alpha de crombach para el total de la prueba es de 0.92 La escala de maltrato del alumno al profesor consta de 15 elementos, que según el análisis factorial se distribuyen en cuatro factores: 1) rechazo-comportamiento disruptivo ("con mi mal comportamiento le impido dar clase", "me desentiendo de lo que dice en clase", "le hablo con malos modales", "le rechazo", le ignoro"; 2) agresión "le agredo físicamente"; "he amenazado a mi profesor", "he roto alguna de sus cosas", "le ridiculizo"; 3 ) antipatía ("algún profesor me resulta antipático", "he pensado que algún profesor no debería estar en este instituto"); 4) maltrato emocional: "me burlo del profesor", "le grito", "le insulto", "le interrumpo cuando habla"). El coeficiente alpha de crombach para el total de la prueba es de .89 .

\section{Procedimiento}

El proceso de evaluación se realizó en dos sesiones, mediante una aplicación colectiva, en la que estuvo presente un solo evaluador. Se especificó que la par- ticipación era voluntaria, garantizándose la confidencialidad de la información. Los padres firmaron una carta para autorizar la participación de sus hijos en el estudio.

\section{RESULTADOS}

Con el objetivo de conocer los distintos tipos de adolescentes respecto a la participación en situaciones de bullying entre compañeros y maltrato con el profesorado, se realizaron análisis de conglomerados mediante el procedimiento de K-Medias. Las tipologías se realizaron para los casos de víctimas y bullies en dos circunstancias: en la interacción alumno-alumno y en la interacción profesor-alumno. Se examinaron diversas soluciones, pero en todos los casos se eligió la de tres conglomerados, ya que aportaba mayor información sobre el tipo de adolescentes identificados para cada tipología.

Tipos de adolescentes en bullying entre iguales (Cluster 1)

En la Tabla 1 se incluyen las medias de los conglomerados del análisis de cluster sobre la participación como víctima y agresor del bullying entre iguales.

Tabla 1. Medias de los conglomerados (tipología) en las situaciones de bullying entre compañeros

\begin{tabular}{|c|c|c|c|}
\cline { 2 - 4 } \multicolumn{1}{c|}{} & \multicolumn{3}{c|}{ Conglomerado } \\
\cline { 2 - 4 } \multicolumn{1}{c|}{} & $\begin{array}{c}\text { Bullies } \\
\text { (1) }\end{array}$ & $\begin{array}{c}\text { Víctima } \\
(2)\end{array}$ & $\begin{array}{c}\text { No involucrado } \\
\text { (3) }\end{array}$ \\
\hline Víctima de compañeros & & 11.05 & 6.16 \\
\hline Víctima de exclusión & 6.80 & 10.44 & 6.81 \\
\hline Víctima de agresión media & 7.38 & 5.35 & 4.08 \\
\hline Víctima de agresión extrema & 4.13 & & 8.59 \\
\hline Agresor hacia compañeros & & 10.95 & 8.10 \\
\hline Agresor de exclusión y gravedad media & 14.42 & 8.86 & \\
\hline Agresión grave & 9.02 & & \\
\hline
\end{tabular}


La solución adoptada permite diferenciar tres tipos de adolescentes respecto a la participación en el bullying entre iguales:

Grupo 1. Bullies. La principal diferencia de este grupo con los otros dos es la mayor frecuencia con la que participan excluyendo a sus compañeros y agrediéndolos en situaciones de gravedad media (con agresiones de tipo psicológico), también puntúan más alto que los otros dos, sobre todo que el grupo tres, en: agresiones graves, y en ser víctimas tanto de agresiones de gravedad media como extrema. Es decir que lo distintivo en ellos es su participación como agresores, aunque esta situación parece ir acompañada de un poco más de riesgo de ser víctimas que el que sufre el grupo 3, no involucrado. Este grupo está conformado por 123 adolescentes, representa el $16.3 \%$ del total de participantes, de ellos 80 son hombres, $10.6 \%$ del total (representan el 65\% de este grupo y el 19.6\% del total de hombres), y 43 son mujeres, que conforman el $5.7 \%$ del total de participantes (representan el 35\% de este grupo y el $12.4 \%$ de las mujeres).

Grupo 2. Víctimas. Este grupo está compuesto por alumnado que se diferencia de los otros dos por ser, sobre todo, víctima tanto de exclusiones como de agresiones de gravedad media y extrema por parte de los compañeros. Conviene tener en cuenta que la frecuencia con la que los miembros de este grupo par- ticipan en exclusiones y agresiones de gravedad media es mayor que la del grupo tres, no involucrado, aunque mucho menor que la del grupo uno, de agresores. Diferencias similares pero de menor magnitud se observan respecto a la agresión extrema. Este grupo está formado por 64 adolescentes, representa el 8.5\% del total de participantes; 36 de ellos son hombres, $4.8 \%$ del total de adolescentes participantes (representan el $56.3 \%$ de este grupo y el $8.8 \%$ del total de hombres), y 28 son mujeres, conforman el 3.7\% del total de participantes (representan el $43.8 \%$ de este grupo y el $8.1 \%$ del total de mujeres).

Grupo 3. No involucrados. Este grupo está formado por 567 adolescentes que no participan apenas en situaciones de violencia escolar, ni como víctimas ni como agresores. Representa el 75.2\% del total de adolescentes participantes, de ellos, 292 son hombres, y conforman el 38.7\% del total de (representan el 51.5\% de este grupo y el $71.6 \%$ del total de hombres participantes), y 275 son mujeres, el $36.5 \%$ del total (representa el $48.5 \%$ de este grupo y el $79.5 \%$ del total de las mujeres participantes).

El análisis de varianza puso de relieve que todas las situaciones de violencia escolar entre iguales (víctima, agresor y no involucrado), contribuyen significativamente a la tipología $(\mathrm{p}<.001)$.

Figura 1. Medias de los tres conglomerados de la situación de bullying en la interacción alumno-alumno

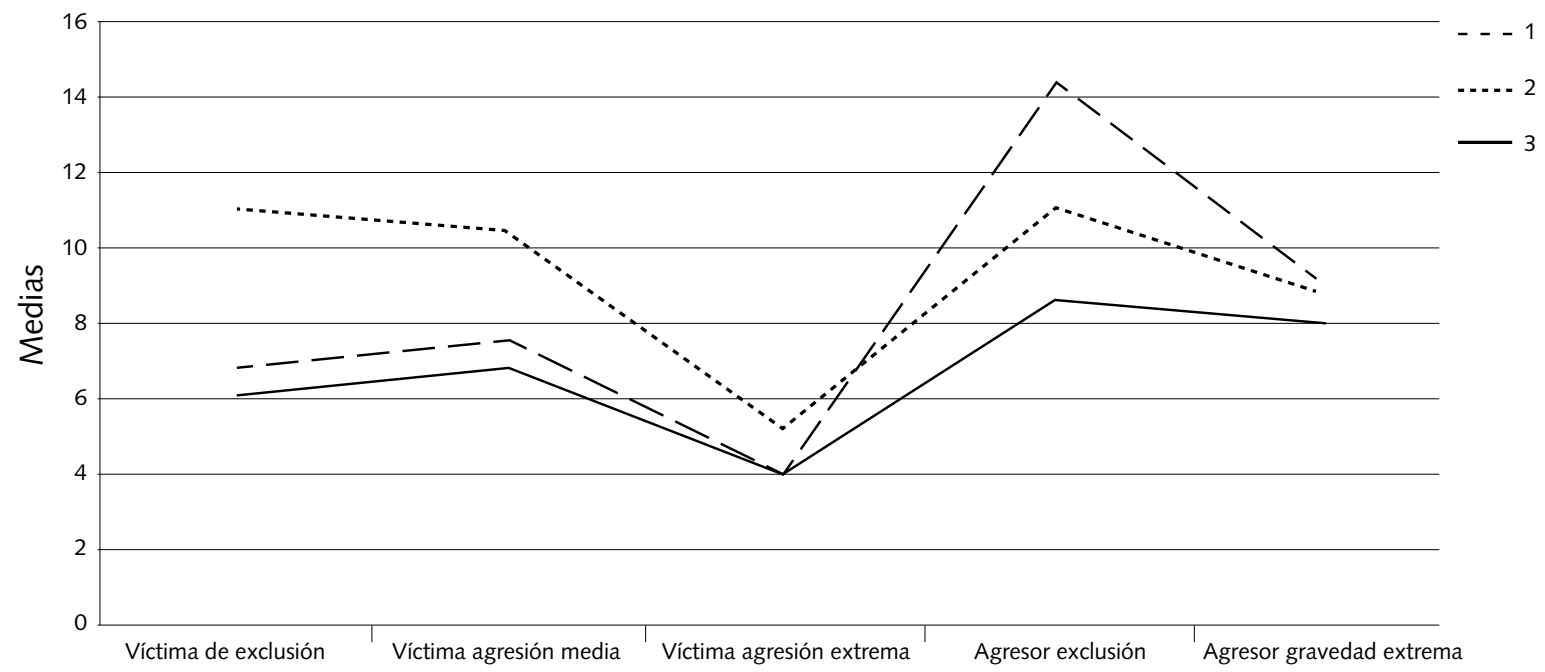


En la figura 1 (página anterior) se observan gráficamente los tres grupos identificados en la tipología de adolescentes que participan en situaciones de bullying en la relación con sus compañeros. Los grupos identificados son: 1: agresores; 2: víctimas; y 3: no involucrados.

Tipos de adolescentes respecto a la interacción profesores-alumnos (Cluster 2)

En la Tabla 2 se incluyen los resultados del análisis de las medias de los conglomerados para definir la tipología de participación de los estudiantes en el maltrato en la interacción con los profesores, contemplando tanto las situaciones en las que el alumno es la víctima, como aquellas en las lo es el profesor.

La solución adoptada con respecto a la tipología de situaciones de maltrato en la interacción alumnoprofesor permite diferenciar los tres grupos siguientes.

Grupo 1.Desafección. Este grupo está compuesto por alumnado que afirma recibir trato discriminatorio y exclusión por parte del profesorado, y expresar antipatía, rechazo y comportamiento disruptivo hacia éste.
El grupo está formado por 178 adolescentes que representan el 23.7\% del total de participantes; de ellos, 106 son hombres, conforman el $14 \%$ del total de participantes (representan el $59.6 \%$ de este grupo y el $26 \%$ del total de hombres participantes), y 72 son mujeres, el 9.6\% del total de participantes (representan el $40.4 \%$ de este grupo y el $20.9 \%$ de las mujeres participantes).

Grupo 2. Acoso. Este grupo está compuesto por alumnado que afirma recibir del profesor trato discriminatorio, agresión, exclusión y maltrato emocional) y expresarle (a éste) antipatía, rechazo, agresión y maltrato emocional. Este grupo está formado por 45 adolescentes, el $6 \%$ del total de participantes, 26 son hombres, (3.5\% del total de adolescentes participantes, representan el $57.8 \%$ de este grupo y el $6.4 \%$ del total de hombres participantes), y 19 son mujeres ( $2.5 \%$ del total de participantes, representan el $42.2 \%$ de este grupo y el 5.5\% del total de mujeres participantes).

Grupo 3. Neutro. Este grupo está formado por alumnado que no se involucra en situaciones de disrupción ni acoso en la interacción con el profesorado.

Tabla 2. Medias de los conglomerados en las situaciones de maltrato en la interacción con el profesorado

\begin{tabular}{|c|c|c|c|}
\cline { 3 - 4 } \multicolumn{2}{c}{} & \multicolumn{2}{c|}{ Conglomerado } \\
\cline { 2 - 4 } \multicolumn{1}{c|}{} & Desafección & Acoso & \multicolumn{2}{c|}{ Neutro } \\
\hline PERCEPCIÓN DE SER VÍCTIMA DEL PROFESOR & & 6.51 \\
\hline Trato discriminatorio & 10.75 & 14.53 & 4.15 \\
\hline Agresión & 4.79 & 7.18 & 4.57 \\
\hline Exclusión & 6.36 & 11.18 & 3.27 \\
\hline Maltrato emocional & 4.23 & 6.80 & \\
\hline PERCEPCIÓN DE VICTIMIZAR AL PROFESOR & & & 5.79 \\
\hline Rechazo y comportamiento disruptivo & 8.14 & 12.20 & 4.07 \\
\hline Agresión & 4.46 & 6.49 & 3.49 \\
\hline Antipatía & 5.70 & 7.13 & 4.93 \\
\hline
\end{tabular}


Son 530 adolescentes, que representan el $70.3 \%$ del total de participantes; 275 son hombres (el 36.6\% del total de participantes, representan el 52\% de este grupo y el $67.6 \%$ de los hombres), y 254 son mujeres (el 33.8\% del total de participantes, que representa el $48 \%$ de este grupo y el $73.6 \%$ del total de las mujeres participantes). El análisis de varianza puso de relieve que los tres tipos de adolescentes en la situación de maltrato en la interacción profesorado-alumnado, contribuyen significativamente a la definición de la tipología $(\mathrm{p}<.001)$.

En la figura 2 se observan gráficamente los tres grupos identificados en la tipología de adolescentes respecto al maltrato profesor-alumno. Éstos son: grupo 1, desafección; grupo 2, acoso; grupo 3, sin problemas.

\section{Relación entre el acoso entre escolares y los problemas en la interacción con los profesores}

Para conocer si existe una conexión entre el bullying entre compañeros (Cluster 1) y los problemas en la interacción alumno-profesor (Cluster 2), se calcularon los residuos corregidos y el estadístico $X^{2}$. El cálculo de residuos corregidos reflejó que el tipo de adolescentes denominado bullies (en la interacción con sus iguales, Cluster 1 está sobre representado en el grupo que tiene problemas de desafección y rechazo en la relación con los profesores, (residuo corregido 3.0), y en el grupo que tienen problemas de acoso y maltrato, con un valor estadístico de 65,590 (p<.001) y un valor de coeficiente de V de Cramer de 0.209. En la figura 3 se observan dichas relaciones. Conviene tener en cuenta que el grupo que actúa como bullies de sus compañeros, que representa el $16.2 \%$ del total, es el $51.1 \%$ de los que participan en escalamiento agresivo (quienes reportan ejercer y recibir maltrato en la relación) con el profesorado (residuo corregido 6.5) y el $23 \%$ de los que manifiesta desafección y comportamiento disruptivo.

Conviene destacar que los estudiantes que se denominan no involucrados en situaciones de bullying con sus pares como víctimas o bullies están sobre representados entre aquellos de los que tampoco tienen problemas en su relación con el profesorado (identificados como estudiantes sin involucramiento) (residuo corregido 6.5).

Figura 2. Medias de los tres conglomerados de las situaciones de maltrato en la interacción profesor-alumno

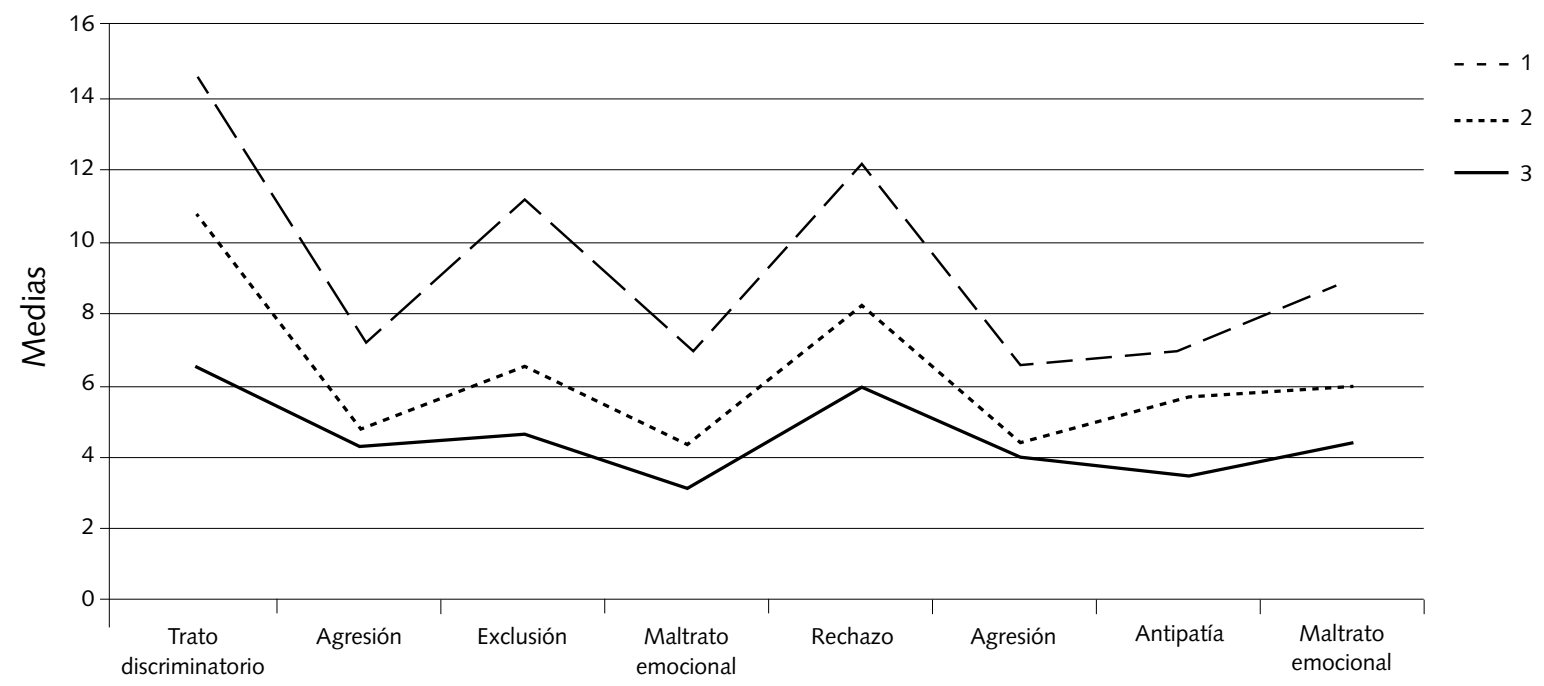


Figura 3. Tipología de la situación de bullying entre iguales y su relación con el maltrato que reciben del profesor

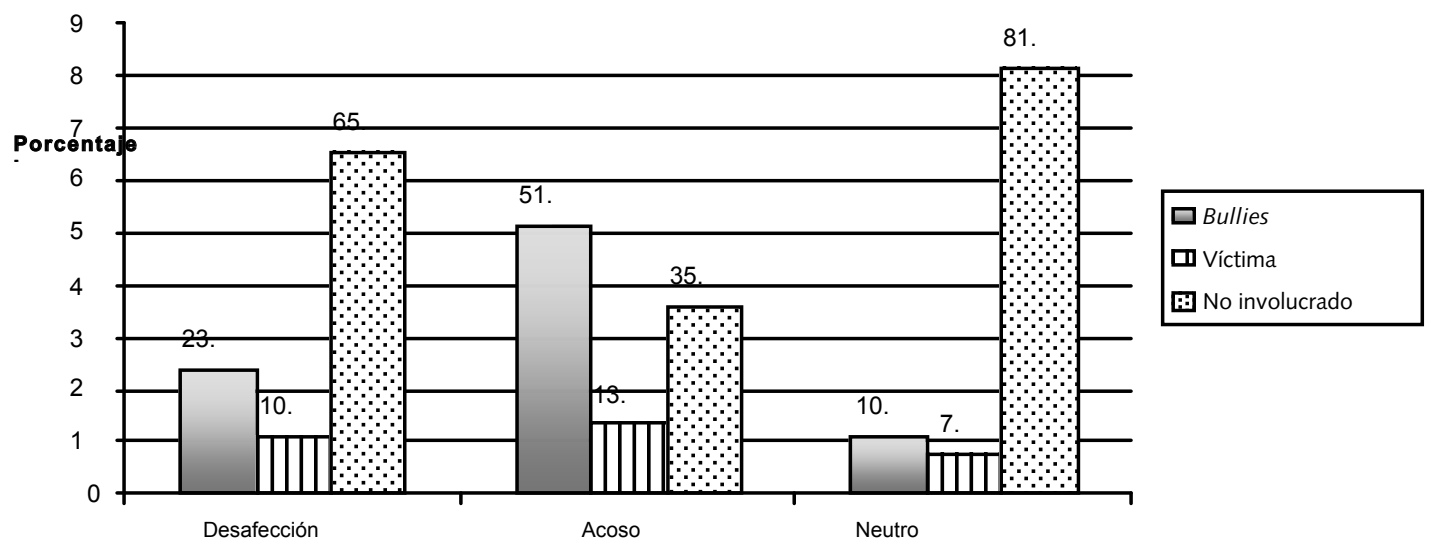

\section{DISCUSIÓN}

La investigación sobre el bullying entre escolares realizada durante los últimos años ha contribuido a superar el tabú que impedía reconocerlo y tratar de erradicarlo. Los resultados obtenidos en esta investigación reflejan la posibilidad y conveniencia de extender la metodología utilizada para detectar dicho bullying al estudio de los problemas existentes con los profesores, reconociendo que el maltrato puede producirse no sólo desde el profesor al alumno, como encuentran Benbenisthy, et al., (2002) en Israel, y Delfabbro, et al., (2006) en Australia, sino también desde el alumno al profesor, como se observa en el estudio de Terry (1998) realizado en el Reino Unido.

Los resultados de este estudio permiten sugerir la integración de otro participante al ciclo de bullying presentado por Olweus (2005); éste sería el noveno participante: el profesorado, que sin duda interviene en este ciclo, ya sea como un agente de cambio que puede detener los episodios de bullying entre pares, como víctima (Terry, 1998) o como agresor (Delfabbro, et al., 2006; Mendoza, 2006, 2009).

El análisis de cluster sobre el bullying entre iguales llevado a cabo en este estudio ha permitido detectar, en la dirección de lo obtenido en investigaciones anteriores, tres situaciones diferentes: la de los que no participan (el 75.2\%); la de quienes participan predominantemente como agresores (un 16.3\%), entre quienes quizá están los que dirigen la agresión y los que les siguen, y la de quienes intervienen sobre todo como víctimas (el 8.5\%). A diferencia de lo obtenido en algunos tra- bajos anteriores (Pelligrini, Bartini, \& Brooks, 1999; Schwartz, Procto \& Chien, 2001; Solberg \& Olweus, 2003), en esta investigación no aparece diferenciado un cuatro grupo específico de agresores-victimas, sino cierta tendencia de los bullies a reconocer que reciben agresiones, relacionada quizá con su orientación a justificar el acoso que ejercen en función de las reales o supuestas ofensas que sufren, y cierta tendencia en las víctimas a ignorar y agredir, que cabe atribuir a la tendencia a reaccionar con hostilidad ante las agresiones recibidas. Parece que el contexto influye sobre todo en la forma en la que se combinan los dos papeles de víctima y agresor en determinados alumnos, puesto que en la mayoría de los trabajos anteriores se había detectado dentro del grupo de víctimas (Schwartz, et al., 2001), en algunos con características más parecidas a los bullies (Unnover, 2005), y aquí en ambos papeles, pero sin impedir que aparezca claramente uno como predominante. El análisis sobre los problemas de interacción con los profesores desde el punto de vista del alumno ha permitido detectar dos tipos de problemas de extensión, naturaleza y gravedad claramente diferentes. En ambos parece existir una estrecha relación entre el trato que el alumno recibe de los profesores y su conducta hacia ellos. El problema más extendido, que afecta a un $23.7 \%$ de estudiantes, gira en torno a lo que perciben como falta de oportunidades para vincularse académicamente con sus profesores, desmotivación y comportamiento disruptivo (que llega incluso a impedir dar clase). Problema que parece de 
naturaleza reactiva y que no cabe considerar maltrato ni acoso. La comparación de estos resultados con los obtenidos en España cuando se pregunta a los profesores de Educación Secundaria (Defensor del Pueblo, 2007) refleja que también destacan como el problema más frecuente "el hecho de que el alumno no deje dar clase". Aunque tal como concluyen Miller, Fergusson y Byrne (2000), las atribuciones de dicho comportamiento por profesores y estudiantes suelen ser muy diferentes. Y como observan Reynard y Sonuga-Barke, (2005), también los estudiantes evaluados aquí lo relacionan con la falta de apoyo por parte del profesor. Apoyo que convendría mejorar en las intervenciones destinadas a erradicar dicho problema, favoreciendo un vínculo de calidad basado en la percepción de la disponibilidad del profesor para conseguir objetivos deseados por los alumnos y ayudarles a obtener protagonismo positivo en el aula.

Teniendo en cuenta el punto de vista de los alumnos, los problemas más graves de interacción con profesores, que afectan al 6\% de los estudiantes, sí podrían ser conceptualizados como maltrato y en algunos casos como bullying. En función de la estrecha relación que se observa aquí entre el trato coercitivo que estos alumnos perciben recibir y dar a sus profesores (con insultos, humillaciones, amenazas), cabe plantear como hipótesis que probablemente se inicien de forma reactiva y se agraven como una conducta instrumental destinada a demostrar el propio poder a la otra parte, que el profesor justificaría aludiendo a la necesidad de disciplina y los alumnos como una defensa ante los abusos que perciben recibir del profesor. Conviene tener en cuenta que la mayoría de los alumnos que participan de estas situaciones también acosan a sus compañeros.

Por otra parte, la fuerte orientación al dominio y al acoso observada en el perfil de este tipo de alumnos con anterioridad (Olweus, 1993) lleva a pensar en la posibilidad de que los episodios coercitivos con sus profesores puedan tener también una función instrumental de demostración del poder, aunque quizá se inicien de forma reactiva, como las escaladas de maltrato detectadas en otros contextos entre adolescentes y adultos (Patterson, 1982). Para poder definir estas situaciones como acoso es necesaria más información de la disponible en este estudio, sobre la naturaleza de los episodios de maltrato recibidos y ejercidos, y la distribución del poder existente en ellos, conociendo, por ejemplo, si el maltrato lo dirigen y lo sufren con los mismos o con diferentes profesores, y por su puesto tomando en cuenta el punto de vista del profesorado.

La comparación de los problemas con los compañeros y con los profesores confirman la existencia de una estrecha relación, que se refleja en el hecho de que el $75 \%$ de los que agreden a compañeros describan problemas con profesores, que en el $51 \%$ de los casos implican maltrato y en el $23.6 \%$ desafección y comportamiento disruptivo. Este resultado pone de manifiesto la necesidad de plantear el estudio y la prevención de la violencia y acoso escolar desde una perspectiva integral que incluya también, como proponen diversos autores (Hyman \& Perone, 1998; Benbenisthy, et al., 2002; Delfabbro, et al., 2006) la victimización que algunos profesores ejercen sobre algunos alumnos; así como la que éstos ejercen contra los profesores, tema mucho menos estudiado (Terry, 1998). Cabe esperar que al ver recogidas sus principales preocupaciones, este tipo de programas venzan las resistencias detectadas en los profesores para participar en la programas de prevención de la violencia cuando se dirigen sólo al bullying entre iguales (Cowie, 2000), relacionada con la tendencia a creer que no es su problema sobre todo en educación secundaria (Behre, Astor \& Meyer, 2001).

Conviene tener en cuenta que en el complejo fenómeno de bullying debe de estudiarse desde una forma más integral en la que no se limite al estudio de las variables que influyen en el proceso de bullying entre pares, sino también el estudio de las variables que producen, mantienen o inhiben el proceso de reciprocidad coercitiva con el profesorado, como el evidenciado en el presente estudio.

En otras investigaciones que se han aproximado al estudio de bullying en la interacción profesor-alumno, se ha identificado que el alumnado expresa que el comportamiento injusto del profesor (Miller, et al., 2000), los elevados niveles de crítica que reciben del profesorado (Daley, Renyard \& Sonuga-Barke, 2005), la rigidez de la clase, e incluso la presión que reciben del grupo de amigos para unirse a la pandilla (Miller, et al., 2000), influyen para que se incremente la conducta negativa en el aula escolar. En contraste, la conducta disruptiva del alumnado es una de las principales fuentes de es- 
trés para los profesores en el aula, aunado a ello que las características del niño, como género, grupo étnico, edad, nivel de competencia, características conductuales, guían el estilo de instrucción del profesorado, que en ocasiones se caracteriza por niveles elevados de crítica y conflicto (Daley, Renyard \& Sonuga-Barke, 2005); de esta manera parece originarse un círculo negativo que peligrosamente puede conducir a escaladas de comportamiento coercitivo entre alumnado y profesorado. Sin duda alguna, el profesorado es un agente de cambio para el alumnado, cuyas estrategias didácticas y de control conductual en el aula escolar influyen notablemente en la calidad del aprendizaje de éste, por lo que en educación, la agresión por parte del profesorado es un comportamiento inaceptable, como lo señala la Ley General de Educación en México. Por otra parte, la agresión que ejercen algunos alumnos

\section{REFERENCIAS}

Andreou, E. (2001). Bully/victim problems and their association with coping behaviour in conflictual peer interactions among school-age children. Educational Psychology, 21, 59-66.

Austin, S. \& Joseph, S. (1996). Assessment of bully/victim problems in 8 to 11 year-olds. British Journal of Educational Psychology, 66, 447-456.

Benbenisthy, R., Zeira, A. \& Astor, R. (2002). Children's reports of emotional, physical and sexual maltreatment by educational staff in Israel. Child Abuse and Neglect, 26, 763.782.

Behre, W., Astor, R., \& Meyer, H. (2001). Teachers' reasonning about intervening in school violence: an examination of violence-prone school subcontexts, Journal of Moral Education, 2,131-153.

Boulton, M. J. \& Smith, P. K. (1994). Bully/victim problems in middle-school children: Stability, self-perceived competence, peer perceptions, and peer acceptance. British Journal of Developmental Psychology, 12, 315329.

Brophy ,J. (1985). Teacher-student interaction. En: Dusek,J. (Ed.) Teacher expectancies. New Jersey: Lawrence Erlbaum Associates.

Bru, E., Stephens, P. \& Tosheim, T. (2002). Students' perceptions of class management and reports of their hacia los profesores es un comportamiento que no se debe permitir; hoy día existen intervenciones probadas científicamente para ayudar a niños y adolescentes a controlar su enojo, enseñándoles a resolver situaciones conflictivas sin agresión (Mendoza, 2010).

Finalmente, los resultados aquí demostrados aportan evidencia sobre el proceso de reciprocidad que existe en los intercambios coercitivos en la relación profesorado-alumnado, que funcionan como un facilitador de escalamiento de agresión.

Se hace necesario que en futuros trabajos de investigación se recoja también la percepción del profesorado, con la finalidad de tener una visión integral que permitea conocer la incidencia del bullying en la interacción profesorado-alumnado desde la opinión de uno de los agentes de cambio más importantes en la comunidad educativa: el profesorado.

own misbehavior. Journal of School Psychology, 40,4, 287-307.

Cowie, H. (2000). Bystanding or standing by: Gender issues in coping with bullying inschools. Aggressive behavior, 26, 85-97.

Daley, D., Renyard, R. \& Sonuga-Barke, E. J.S. (2005). Expressed emotion in teachers of children with and without behavioural difficulties. British Journal of Educational Psychology, 75, 25, 35.

Defensor del Pueblo (2007). Violencia escolar: el maltrato entre iguales en la Educación Secundaria Obligatoria. Madrid: Publicaciones del Defensor del Pueblo.

Delfabbro, P., Winefield, T., Trainor, S., Dollard, M., Anderson, S., Metzer, J. \& Hammarstrom, A. (2006). Peer and teacher bullying/victimization of South Australian secondary school students: Prevalence and psychosocial profiles. British Journal of Educational Psychology, 76, 71-90.

Díaz-Aguado, M.J., Martínez Arias, R. \& Martín Seoane, G. (2004). Prevención de la Violencia y lucha contra la exclusion desde la adolescencia: La violencia entre iguales en la escuela y el ocio. Volumen 2: Programa de intervención y estudio experimental. Madrid: INJUVE 
Eslea, M., Menesini, E., Morita, Y., O'Moore, M., MoraMerchan, J., Pereira, B., Smith, P. K. \& Zhang, W. (2003). Friendship and Loneliness among Bullies and Victims: Data From Seven Countries. Aggressive Behavior. 30, 71-83.

Hawker, D. S. J. \& Boulton, M.J. (2000). Twenty years research on peer victimization and psychosocial maladjustment: A meta-analytic review of crosssectional studies. Journal of Child Psychiatry and Psychiatry, 41, 441-455.

Hyman, I. \& Perone, D. (1998). The other side of school violence: Educator policies and practices that may contribute to sudent misbehavior. Journal of School Psychology, 36,1, 7-27.

Ireland, J., \& Power, Ch. (2004). Attachment, Emotional Loneliness, and Bullying Behaviour: A Study of Adult and Young Offenders. Aggressive Behaviour, 30, 298 312.

Kokkinos, C. \& Panayiotou, G. (2004). Predicting bullying and victimization among early adolescents: Associations with disruptive behaviour disorders. Aggressive Behaviour, 30, 520-533.

Lewis, R. (2001). Classroom discipline and student responsibility: The students'view. Teaching and teacher education, 17, 307-319.

Ma, X. (2002). Bullying in Middle School: Individual and school characteristics of victims and offenders. School Effectiveness and School Improvement, 13, 1, 63-89.

Mendoza, B. (2006). Las dos caras de la violencia escolar: El maltrato en la interacción profesor-alumno y entre iguales. Tesis Doctoral. Universidad Complutense de Madrid, España.

Mendoza, B. \& Santoyo, C. (2008). Trayectorias de desarrollo del fenómeno de acoso escolar (bullying). Ponencia presentada en el Segundo Congreso Internacional de Psicología del Desarrollo. Psicología. Universidad Autónoma de Aguascalientes, México.

Mendoza, B. (2009). Taller para la Detección de Casos de Abuso Sexual Infantil en Niños de Educación Básica. Revista Iberoamericana de Psicología. 17, 1,23-36.

Mendoza, B. (2010). Manual de Auto control de enojo. Tratamiento Cognitivo-Conductual. México: El Manual Moderno.

Mendoza, B. \& Santoyo, C. (2010). Evaluación Conductual del desequilibrio de poder de intercambios socia- les asociados al bullying. Ponencia presentada en el Tercer Seminario Bienal sobre Desarrollo e Interacción Social: Aristas y Perspectivas Múltiples. Facultad de Psicología. UNAM.

Miller, A., Ferguson, E. \& Byrne, I. (2000). Pulils'causal attributions for difficult classroom behavior. British Journal of Educational Psychology, 70, 85-96.

Monks, C. \& Smith, P. K. (2000). Relations of children involved in bully-victim problems at school. In R.S.L. Mills y S. Duck (Eds), Developmental psychology of personal relationships. Chichester: Wiley and Sons.

Natvig, G., Albrektsen, G. \& Qvarnstrom, U. (2001) School-related stress experience as a risk factor bor bullying behavior. Journal of Youth and Adolescence, $30,5,561-575$.

Olweus, D. (1978). Aggression in the schools: Bullies and whipping boys. Washington, DC: Hemisphere Wiley.

Olweus, D. (1993). Bullying at School: What we know and what we can do. Oxford: Blackwell.

Olweus, D (2001). Bullying at school: tacling the problem. Observer, 225, 24-26.

Olweus, D. (2005). Bullying en la escuela: datos e intervención. Documento Presentado en laIX Reunión Internacional sobre biología y sociología de la violencia. Violencia y la Escuela. Valencia, España.

Patterson, G. (1982). Coercitive family process. Oregon: Eugene.

Pellegrini, A., Bartini, M. \& Brooks, F. (1999) School bullies, victims and aggressive victims. Journal of Educational Psychology, 91, 216-224.

Reynard, D. \& Sonuga-Barke, E. (2005). Teachers emotional expression about disruptive boys. British Journal of Educational Psychology, 75, 1, 25-35.

Salmivalli, C.; Lagerspetz, M.; Björkkqvist, K.; Österman, D. \& Kauklainen, A. (1996). Bullying as a group process. Aggressive Behavior, 22, 1-15.

Salmivalli, C., Lappalainen, M. \& Lagerspetz, M. (1998). Stability and change of behavior in connection with bullying in schools: A two year follow up. Aggressive Behavior, 24, 205-218.

Santoyo, C., Colmenares, L., Figueroa, N., Cruz, A. \& López, E. (2008). Organización del comportamiento coercitivo en niños de primaria: un enfoque de síntesis. Revista Mexicana de Psicología, 25,1, 71-87. 
Schwartz, D., Proctor, I.J., Chien, D.H., (2001). The aggressive victim of bullying: Emotional and behavioural dysregulation as a pathway to victimization by peers. En: Juvonen, J., y Graham S, editors. Peer harassment in schools. New York: Guilford Press.

Solberg,M.; Olweus, D. (2003). Prevalence estimation of school bullying with the Olweus bully/victim questionnaire. Aggressive Behaviour, 29, 239-268.

Slee, P.T. \& Rigby, K. (1993). The relationship of Eysenck's personality factors and self-steem to bully/victim behaviour in Australian school boys. Personality and Individual Differences, 14, 371-373.

Terry, A. (1998). Teachers as targets of bullying by their pupils: a study to investigateincidence. British Journal of Educational Psychology, 68, 255-268.
Unnever, J. (2005). Bullies, Aggressive Victims, and Victims: Are They Distinct Groups? Aggressive Behavior, 31, 153-171.

Wilson, J.W., \& Herrnstein, R.J. (1985). Crime and human nature. New York: Simon \& Schuster, Inc.

Wentzel, K. (2002). Are effective teachers like good parents? Teaching styles and student adjustment in early adolescence. Child Development, 73,1, 287-301.

Young, R. \& Sweeting, H. (2004). Adolescent bullying, relationships, psychological well-being and genderatypical behavior: A gender diagnosticity approach. Sex Roles, 50, 7-8, 525-555. 